\title{
Elastic $p-{ }^{3} \mathrm{He}$ and $\mathrm{n}-{ }^{3} \mathrm{H}$ scattering with two- and three-body forces
}

\author{
B. Pfitzinger and H. M. Hofmann \\ Institut für Theoretische Physik III, University of Erlangen-Nürnberg, Staudtstraße 7, D 91058 Erlangen, Germany \\ G. M. Hale \\ Theoretical Division, Los Alamos National Laboratory, Los Alamos, NM 87545, USA
}

\begin{abstract}
We report on a microscopic calculation of $n-{ }^{3} \mathrm{H}$ and $p-{ }^{3} \mathrm{He}$ scattering employing the Argonne $v_{18}$ and $v_{8}^{\prime}$ nucleon-nucleon potentials with and without additional three-nucleon force. An $R$ matrix analysis of the $p-{ }^{3} \mathrm{He}$ and $n-{ }^{3} \mathrm{H}$ scattering data is presented. Comparisons are made for the phase shifts and a selection of measurements in both scattering systems. Differences between our calculation and the $R$-matrix results or the experimental data can be attributed to only two partial waves $\left({ }^{3} P_{0}\right.$ and $\left.{ }^{3} P_{2}\right)$. We find the effect of the Urbana IX and the Texas-Los Alamos three-nucleon forces on the phase shifts to be negligible.
\end{abstract}

\section{Introduction}

It is well known that realistic nucleon-nucleon $(N N)$ forces cannot reproduce the ${ }^{3} \mathrm{H}$ and ${ }^{3} \mathrm{He}$ binding energies. Three-nucleon interactions (TNIs) are added to give the necessary small corrections but they still fail to reproduce certain properties of the three nucleon system, most notably the $A_{\mathrm{y}}$ analyzing power in $N d$ scattering [1]. Yet the $30 \%$ deviation of $A_{\mathrm{y}}$ can be resolved by tiny changes in the $N d$ scattering phase shifts (on the order of 0.1 degrees [2, 3, 44). Furthermore very many operators can contribute to a TNI and the lack of stringent conditions in the three-nucleon system on the structure of the TNI makes its application to other systems desirable. In 5 it was shown that although a realistic $N N$ force can generally reproduce the ${ }^{4} \mathrm{He}$ system, there remain differences, most notably in the analyzing powers. The intensely studied ${ }^{4} \mathrm{He}$ system [6] is unfortunately very difficult to describe due to the many resonances and the ${ }^{4} \mathrm{He}$ bound state. Therefore we investigate the much simpler systems $p-{ }^{3} \mathrm{He}$ and $n-{ }^{3} \mathrm{H}$ where data exist in the energy range of interest.

We organize the paper in the following way: The next section contains a description of the $R$-matrix analysis of the $p-{ }^{3} \mathrm{He}$ and $n-{ }^{3} \mathrm{H}$ data. After that we discuss the Resonating Group Model (RGM) calculation and the model space used. Then we compare $R$-matrix and RGM results of the phase shifts for various model spaces, and demonstrate the differences for a selection of typical observables. Finally we will discuss the effect of TNIs on the scattering.

\section{R-MATRIX ANALYSIS}

The $R$-matrix analysis of the $T=1$ part of the $A=4$ system began many years ago with an analysis of $p+{ }^{3} \mathrm{He}$ scattering data below $20 \mathrm{MeV}$ incident proton energy [7. Only one solution described all the data included, namely the one with $\delta\left({ }^{3} P_{1}\right)>\delta\left({ }^{1} P_{1}\right)$, giving a $\chi^{2}$ per degree-offreedom value of about 1.23. Later, this solution was reflected to the $n+{ }^{3} \mathrm{H}$ system [8], using a simple energy shift to correct for the short-ranged Coulomb differences between ${ }^{4} \mathrm{Li}$ and ${ }^{4} \mathrm{H}$. This shift of the $R$-matrix eigenenergies $\left(\Delta E_{\lambda}=-0.86 \mathrm{MeV}\right)$ for the ${ }^{4} \mathrm{Li}$ system was adjusted by hand to reproduce approximately the $n-t$ total cross section measurement of Phillips, et al. [9].

More recently, we added to the $p+{ }^{3} \mathrm{He}$ analysis analyzing-power and spin-correlation data measured at energies between 4 and $10 \mathrm{MeV}$ by Alley and Knutson [10]. These high-precision data made small changes in the phase shifts, but did not alter the qualitative nature of the solution, shown as the solid curves in Figs. 1-5. With these data we achieve a $\chi^{2}$ per degree-of-freedom of 1.27 .

Finally, we combined the $p+{ }^{3} \mathrm{He}$ data with the $n-t$ total cross sections of ref. 9 and fitted both reactions simultaneously. The single energy shift used earlier did not give a particularly good fit to the total cross sections, so we allowed the low-lying eigenenergies in the ${ }^{4} \mathrm{H}$ system to adjust separately from those in the ${ }^{4} \mathrm{Li}$ system, while keeping the reduced-width amplitudes the same in both systems. This resulted in a $\chi^{2}$ per degree-of-freedom of 1.59. Most of the increase came from the fit to the $n-t$ total cross sections, which have uncertainties on the order of $0.2 \%$. The $\chi^{2}$ per point of the fit to the $p+{ }^{3} \mathrm{He}$ data increased only from 1.22 to 1.24 . The analysis is based on 1447 data points having proton energies between 1.01 and $19.7 \mathrm{MeV}$ and neutron energies between 0.06 and $20.06 \mathrm{MeV}$, and allows a maximal orbital angular momentum $L_{\max }=4$. The ${ }^{4} \mathrm{Li}$ resonance energies still agree with those of [6] which also contains a brief description of the $R$-matrix method.

\section{RGM AND MODEL SPACE}

We use the Resonating Group Model 11, 12, 13] to compute the scattering in the ${ }^{4} \mathrm{H}$ and ${ }^{4} \mathrm{Li}$ systems using the Kohn-Hulthén variational principle [14]. The main technical problem is the evaluation of the manybody matrix elements in coordinate space. The restriction to a Gaussian basis for the radial dependencies of the wave function allows for a fast and efficient calcu- 
lation of the individual matrix elements 11, 13]. However, to use these techniques the potentials must also be given in terms of Gaussians. In this work we use suitably parametrized versions of the Argonne $v_{18}$ [15] and $v_{8}^{\prime}$ [16] $N N$ potentials and the Urbana IX [16] and Texas - Los Alamos [17] TNIs.

The inclusion of an additional TNI requires an order of magnitude more computing power than the realistic $N N$ forces alone. It is therefore very fortunate that enough data exists at low energies for the comparatively simple isospin $T=1$ systems ${ }^{4} \mathrm{H}$ and ${ }^{4} \mathrm{Li}$ to allow for a comparison between calculation and the experimental data or the $R$-matrix analysis thereof.

In the ${ }^{4} \mathrm{Li}$ system we use a model space with three two-fragment channels, namely the $p-{ }^{3} \mathrm{He}$, the ${ }^{2} \mathrm{H}-(p p)$ and the ${ }^{2} \mathrm{H}(S=0)-(p p)$ channels. The last two are an approximation to the three- and four-body breakup channels that cannot in practice be treated within the RGM. The ${ }^{4} \mathrm{Li}$ is treated as four clusters in the framework of the RGM to allow for the required internal orbital angular momenta of ${ }^{3} \mathrm{He}$ or ${ }^{2} \mathrm{H}$.

For the scattering calculation we include the $S, P$ and some of the $D$ wave contributions to the $J^{\pi}=$ $0^{+}, 1^{+}, 0^{-}, 1^{-}$and $2^{-}$channels. From the $R$-matrix analysis these channels are known to give essentially the experimental data. The full wave function for these channels contains over 100 different spin and orbital angular momentum configurations, hence it is too complicated to be given in detail. To give an impression of the model space we will describe the important structures of ${ }^{3} \mathrm{He}$ as used in the present work.

The dominant spin $S=1 / 2$ configurations of ${ }^{3} \mathrm{He}$ include those without angular momentum and with two $D$ waves coupled to total angular momentum $L=1$. $S=3 / 2$ in turn occurs together with a single $D$ wave on each of the Jacobi coordinates or two $D$ waves coupled to $L=2$. Each of these configurations uses a set of one to three Gaussians whose width parameters were obtained by a non-linear optimization using a genetic algorithm [18]. In this small, 29-dimensional model space we still achieve $-6.37 \mathrm{MeV}$ binding energy, an $\mathrm{rms}$ radius of 1.78 $\mathrm{fm}$ and a $D$ state probability of $7.7 \%$ for the ${ }^{3} \mathrm{He}$ using $\mathrm{A} v_{18}$. This must be compared to $-6.92 \mathrm{MeV}$ known from Faddeev calculations [19] whereas we find $-6.88 \mathrm{MeV}$ in a rather large model space.

This representation of ${ }^{3} \mathrm{He}$, together with a $S=1 / 2$ and $L=2$ configuration (excited state) and the $(p n)-$ ( $p p)$ fragmentations, form the basis of our $p-{ }^{3} \mathrm{He}$ scattering calculation. Once the fragment wave functions are fixed the scattering problem is solved with our RGM code relying on the Kohn-Hulthén variational principle [14]:

$$
\delta\left(\left\langle\Psi_{t}|H-E| \Psi_{t}\right\rangle-\frac{1}{2} a_{l l}\right)=0,
$$

where $a_{i j}$ denotes the reactance matrix.

The model space described above (consisting of two to four physical scattering channels for each $J^{\pi}$ ) is by no means sufficient to find reasonable results. So-called distortion or pseudo-inelastic channels 13 have to be added to improve the description of the wave function within the interaction region. Accordingly, the distortion channels have no asymptotic part.

For practical purposes it is obvious to reuse some of the already calculated matrix elements as additional distortion channels. In that way we include all the positive parity states of the three-nucleon subsystem with $J_{3}^{\pi} \leq 5 / 2^{+}$in our calculation. However, it was recently pointed out by A. Fonseca 20] that states having a negative parity $J_{3}^{-}$in the three-nucleon fragment increase the $n-{ }^{3} \mathrm{H}$ cross section noteably. Therefore we also added the appropriate distortion channels in a similar complexity as in the $J_{3}^{+}$case to our calculation, thereby doubling the size of the model space.

\section{PARTIAL WAVE ANALYSIS}

The $R$-matrix analysis of the $T=1$ part of the $4 N$ system described above represents the currently available $p-{ }^{3} \mathrm{He}$ and $n-t$ data very well, as the solid lines in figs. 2, 3 and 5 show. In addition it provides elastic scattering

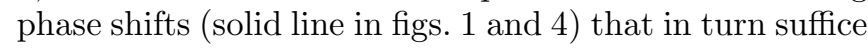
to describe the experimental data. Therefore we take these phase shifts as benchmarks against which to test our calculations.

In a calculation of elastic $p-{ }^{3} \mathrm{He}$ scattering using the full model space described above (including both the $J_{3}^{+}$ and $J_{3}^{-}$distortion channels) we find for both the Argonne $v_{18}$ and $v_{8}^{\prime}$ (not shown) interactions in general phase shifts very similar to those given by the $R$-matrix analysis (see figs. 1 and 1 ). The $S$-waves are negative due to the underlying Pauli-forbidden states, whereas all the $P$-waves are positve. The $J_{3}^{-}$part increases the model space and therefore reduces the repulsion in the $S$-waves and increases the (attractive) interaction in the $P$-waves. Hence, all phase shifts have to become more positive. The ${ }^{1} S_{0}$ and ${ }^{3} S_{1}$ phase shifts (see fig. (1) depend only weakly on the $J_{3}^{-}$part of the model space (long dashed line as compared to dashed line), because the central terms of the $N N$-interaction can only connect to a few states of the increased model space. The ${ }^{3} P_{0}$ and ${ }^{3} P_{2}$ phase shifts deviate markedly from the $R$ matrix results (see fig. 1). Without the $J_{3}^{-}$components (long dashed line) the major difference between our calculation and the $R$-matrix results was the ${ }^{3} P_{2}$ matrix element being too small, whereas the ${ }^{3} P_{0}$ results were very close. The additional $J_{3}^{-}$distortion channels reduce by half the difference for ${ }^{3} P_{2}$, but also raise the ${ }^{3} P_{0}$ phase considerably beyond its $R$-matrix values.

It has already been pointed out previously [5] that the calculated ${ }^{3} P_{2}$ matrix element in the ${ }^{4} \mathrm{He}$ system is too small (then using the realistic Bonn $N N$ interaction [21]). It was shown that the manual change of ${ }^{3} P_{2}$ to its $R$ matrix value removed most of the discrepancies in the description of the analyzing power. The $J_{3}^{-}$part of the $p-{ }^{3} \mathrm{He}$ model space in the present calculation acts mainly 

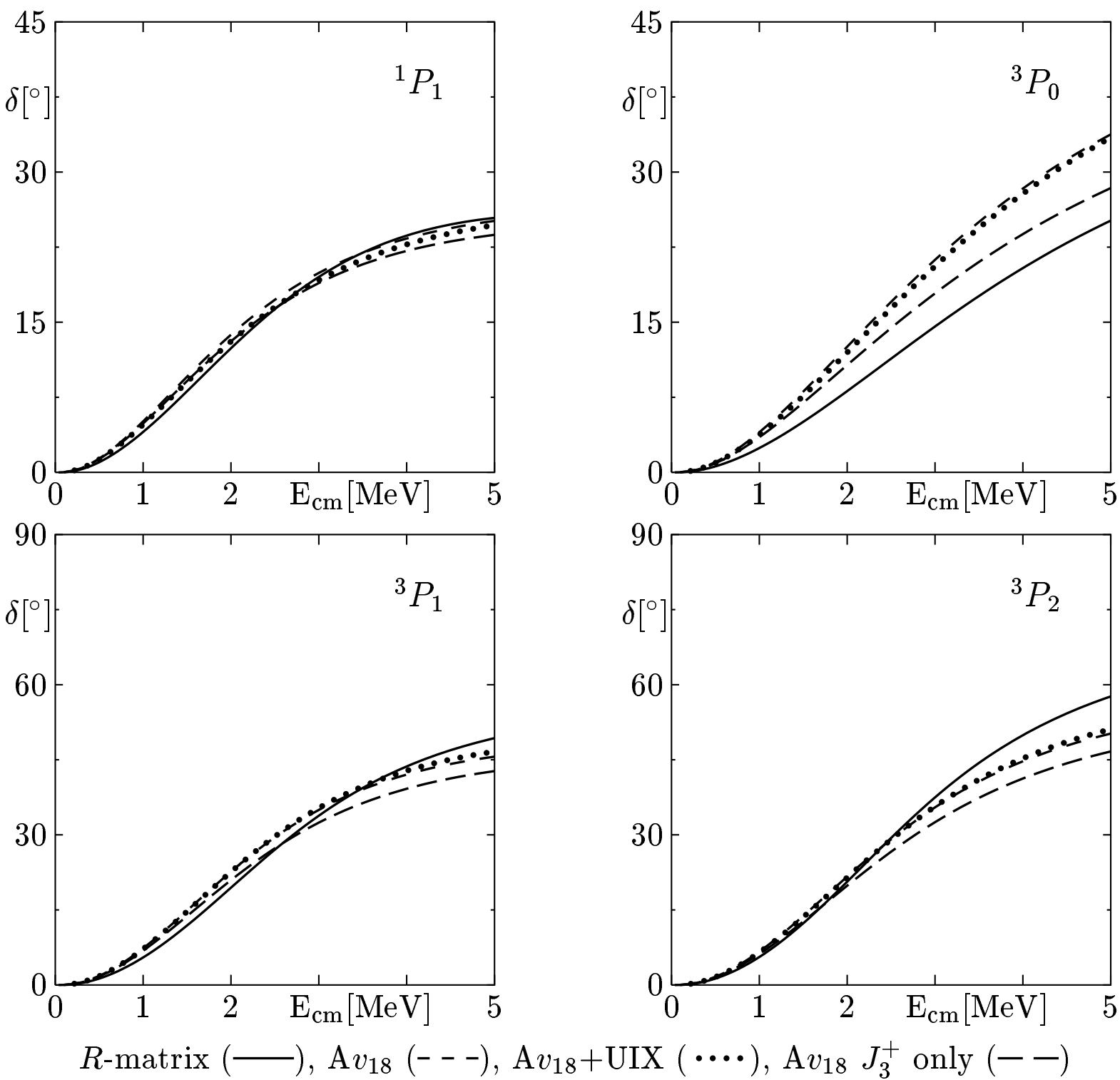

FIG. 1: $\quad p-{ }^{3} \mathrm{He} P$ wave scattering phase shifts. RGM calculations (A $v_{18}$, dashed line, restricted to postive parity in the three-nucleon subsystem, long dashed, and $\mathrm{A} v_{18}+$ Urbana IX, dotted) compared to the $R$-matrix analysis (solid line).

on the ${ }^{3} P_{0}$ and ${ }^{3} P_{2}$ channels increasing both of them by several degrees (fig. 1). However, the splitting of ${ }^{3} P_{2}-$ ${ }^{3} P_{0}$ remains considerably too small, see fig. If where the deviations of the calaculated $p-{ }^{3} \mathrm{He}$ phase shifts from the $R$-matrix values and the ${ }^{3} P_{2}-{ }^{3} P_{0}$ splitting are shown. We note in passing that distortion channels as described above were not included in $[5]$.

\section{COMPARISON WITH DATA}

The increase of the ${ }^{3} P_{2}$ matrix element due to the $J_{3}^{-}$ components suffices to describe the total and differential cross sections (figures 2 and 5 ) in both the $n-{ }^{3} \mathrm{H}$ and $p-{ }^{3}$ He scattering. Again, the improvement is due to the larger ${ }^{3} P_{2}$ matrix element, the effect of ${ }^{3} P_{0}$ being too large on the cross sections is negligible (on the order of $1 \%$ ) because of the small statistical weight.

There remain deviations of the differential cross section at small and large scattering angles as well as in the low energy behavior of the total cross section. In the first case, we attribute the differences at large scattering angles to be an effect of the model space lacking higher partial waves, and our calculation agrees with the results obtained by restricting the $R$-matrix partial waves to those included in the RGM. Even compared to these results, our calculation differs in the region of the interference between the Coulomb and the strong interaction at small scattering angles. This discrepancy cannot be attributed to a single partial wave, but originates from 


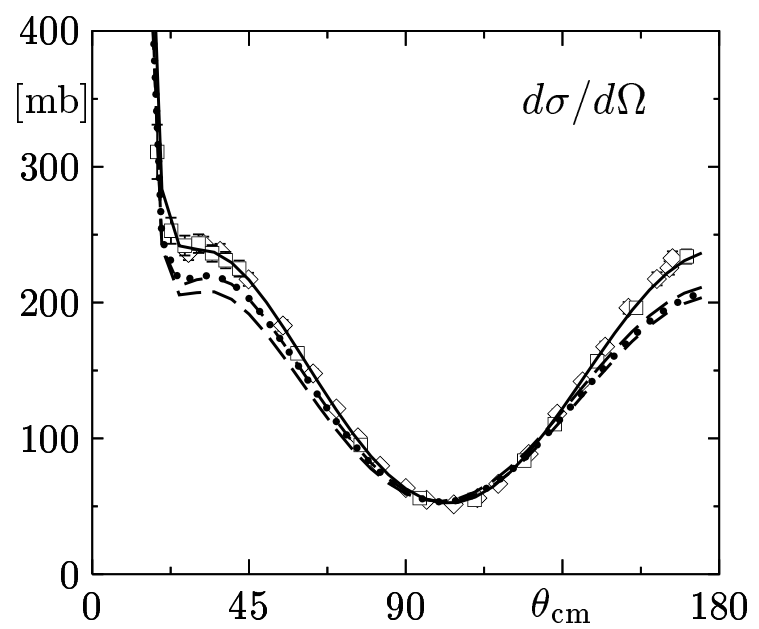

FIG. 2: $\quad p-{ }^{3}$ He differential cross section at $E_{\mathrm{cm}}=4.1 \mathrm{MeV}$. The experimental data is from [22] $(\diamond)$ and [23] ( $\square$ ), the longdashed line denotes the results for the $\mathrm{A} v_{8}^{\prime}+\mathrm{TLA}$ interaction, other lines as in fig. 1 .

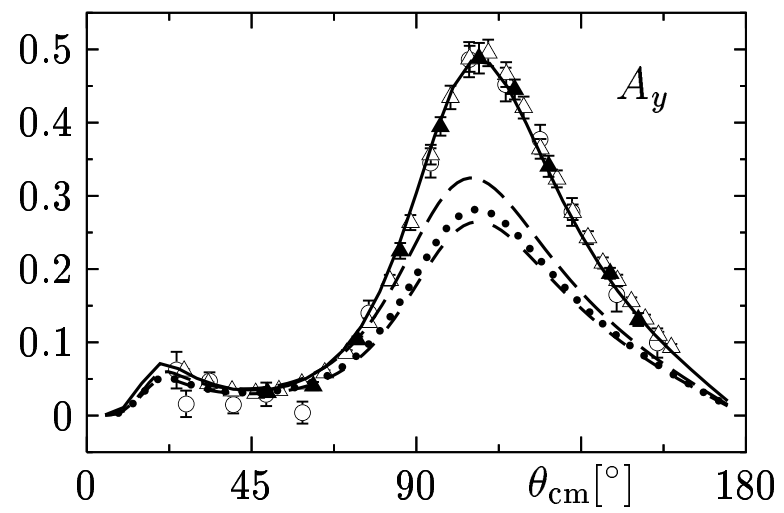

FIG. 3: $\quad p-{ }^{3} \mathrm{He}$ analyzing power $A_{\mathrm{y}}$ at $E_{\mathrm{cm}}=4.1 \mathrm{MeV}$. The experimental data is from [24] (०) and [10] $(\Delta, \mathbf{\Delta})$, the lines are as in fig. 11.

tiny differences between the $R$-matrix phase shifts and the calculated ones.

The total cross section of $n-{ }^{3} \mathrm{H}$ scattering at low energies depends mainly on the ${ }^{3} S_{1}$ phase shift whose statistical weight $2 J+1$ is three compared to one for ${ }^{1} S_{0}$. Small differences in ${ }^{3} S_{1}$ (fig. (4) inset) lead to the $10 \%$ deviation between the different models ( $\mathrm{A} v_{18}$ dashed and long dashed, $J_{3}^{+}$only in fig. 5). Note that the large effect of the Urbana IX TNI at low energies is a combination of the good reproduction of the low energy ${ }^{3} S_{1}$ matrix element and the ${ }^{1} S_{0}$ phase shift being too small.

As in the ${ }^{4} \mathrm{He}$ system, the real challenge lies in the polarization observables, e. g. the $p-{ }^{3} \mathrm{He}$ proton analyzing power $A_{\mathrm{y}}$. Even within the large model space our calculations find only about half the measured $A_{\mathrm{y}}$ (fig. 3) similar to the findings in [20]. We know from [5] that the analyzing power is sensitive to the ${ }^{3} P_{2}$ matrix element and it was surprising that even with the $J_{3}^{-}$distortion channels increasing the ${ }^{3} P_{2}$ phase shift, we cannot improve the description of $A_{\mathrm{y}}$. The reason is that most of the expected improvement due to the larger ${ }^{3} P_{2}$ matrix element is canceled by the increased ${ }^{3} P_{0}$ phase shift that decreases $A_{\mathrm{y}}$, as can be seen when we lower ${ }^{3} P_{0}$ to its $R$ matrix value (fig. 6, where the values of $A_{\mathrm{y}}$ are displayed close to the maximum). Increasing ${ }^{3} P_{2}$ further to the $R$-matrix value will then raise $A_{\mathrm{y}}$ again, but still not to the experimental data. Only a small part of the remaining difference is due to higher partial waves that are not included in the model space (see fig. 6). The remainder comes from small differences in the other partial waves.

\section{THREE-NUCLEON FORCE EFFECTS}

Both the $\mathrm{A} v_{8}^{\prime}$ and $\mathrm{A} v_{18} N N$ forces yield essentially the same phase shifts, and we convinced ourselves that the Gaussian parametrization of the Bonn interaction 21] also agrees with these results. In the previous section we showed that we cannot reproduce the $p-{ }^{3} \mathrm{He}$ analyzing power $A_{\mathrm{y}}$ mainly due to the too small splitting of ${ }^{3} P_{2}$ and ${ }^{3} P_{0}$ (fig. 7). Therefore we included an additional TNI in our calculations. For $\mathrm{A} v_{18}$ we chose the $2 \pi$-exchange model implemented as the Urbana IX force [16] and for $\mathrm{A} v_{8}^{\prime}$ the Texas-Los Alamos (TLA, 17) threenucleon force. The latter includes only short range operators and is claimed to resolve the $A=3 A_{\mathrm{y}}$ problem for a certain choice of its operator strength $\left(c_{1}=3\right.$, [17 $)$. In our calculation we chose this factor to be $c_{1}=1.5$ to give reasonable ${ }^{3} \mathrm{He}$ binding energies in the model space used.

In the case of the $\mathrm{A} v_{18}$ and Urbana IX interaction, the additional TNI leads to the expected improvement of the binding energy. For the small ${ }^{3} \mathrm{He}$ wave function we find $-6.88 \mathrm{MeV}$ instead of the $-6.37 \mathrm{MeV}$ for $\mathrm{A} v_{18}$ alone and the TNI increases the $D$ state probability $P_{D}$ somewhat (from $7.7 \%$ to $8.2 \%$ ). In a rather large model space we find a binding energy of $-7.72 \mathrm{MeV}$ and kinetic energy of $-50.05 \mathrm{MeV}$, close to the values given in [19].

Both TNIs are unfortunately very time-consuming to evaluate and neither of them contributes significantly to the phase shifts (fig. 11). Especially the Urbana IX interaction does not affect the phase shifts at all while still improving the binding energy of ${ }^{3} \mathrm{He}$. The ${ }^{3} P_{2}-{ }^{3} P_{0}$ splitting is somewhat improved by the TLA force but still far from its $R$-matrix values (fig. 7). Yet this improvement can already be seen in the analyzing power (fig. 3), where the calculation including the TLA force gives significantly better results. Since the Urbana force left the phase shifts almost unchanged it affects the analyzing power (fig. 3) only marginally. These effects can be more clearly seen in fig. 6, where the analyzing power close to the maximum is shown for the different models.

Since the $2 \pi$ exchange operators leave the scattering 

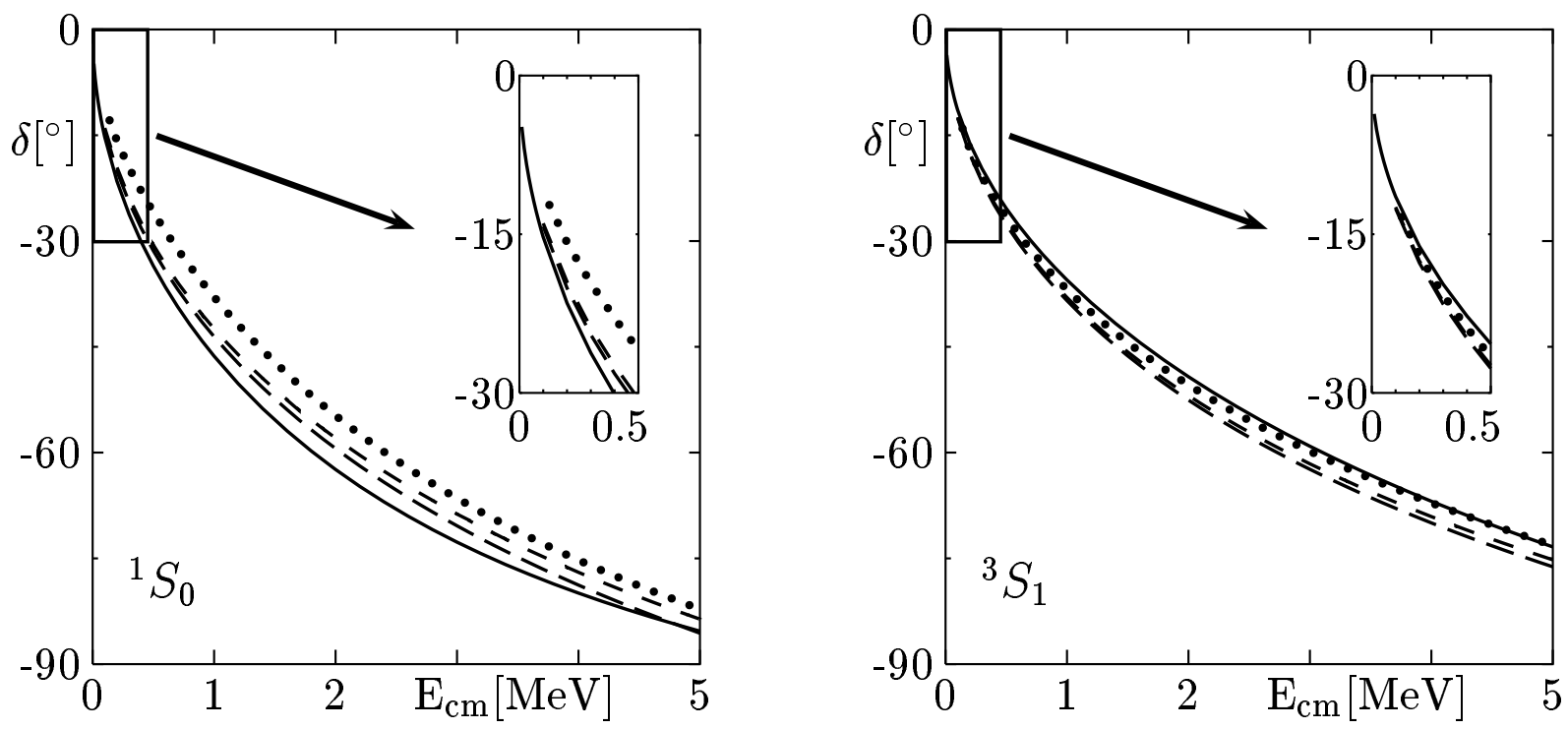

FIG. 4: $S$ wave phase shifts of elastic $n-{ }^{3} \mathrm{H}$ scattering, the lines are as in fig. 1.

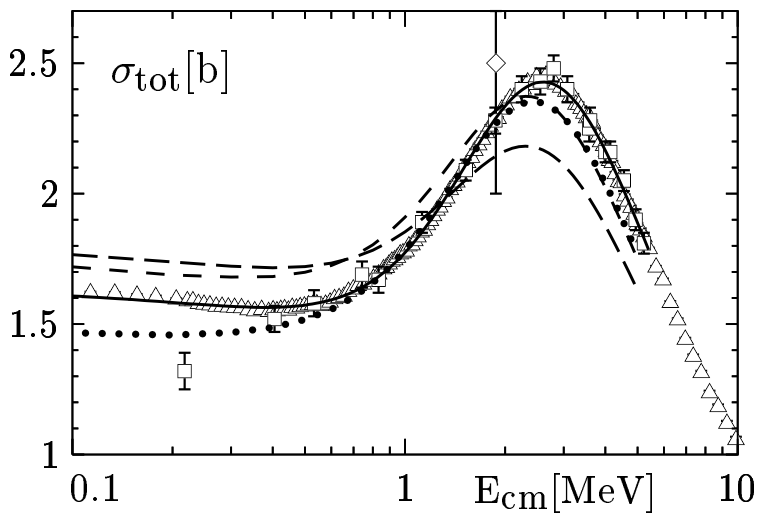

FIG. 5: $n-{ }^{3} \mathrm{H}$ total cross section. The experimental data is from [9] $(\triangle), 25(\square)$ and 26] $(\diamond)$, the lines are as in fig. 1 .

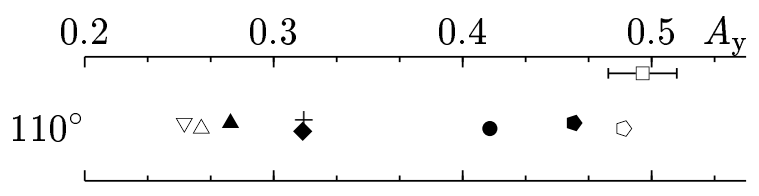

FIG. 6: $\quad p-{ }^{3}$ He proton analyzing power at $E_{\mathrm{cm}}=4.1 \mathrm{MeV}$ and 110 degrees for different models: $\mathrm{A} v_{18}(\triangle), \mathrm{A} v_{18} J_{3}^{+}$ only $(\nabla), \mathrm{A} v_{18}+\operatorname{Urbana} \operatorname{IX}(\boldsymbol{\Delta}), \mathrm{A} v_{8}^{\prime}+\mathrm{TLA}(\bullet), \mathrm{A} v_{18}$ with ${ }^{3} P_{0}(+)$ and with both ${ }^{3} P_{0}$ and ${ }^{3} P_{2}(\bullet)$ adjusted, $R$ matrix (pentagon) and $R$-matrix restricted to $L \leq 2$ (filled pentagon), data point $(\square)$ from $[10]$.

phase shifts unchanged, we considered the $V_{3}^{*}$ operators proposed in 27] in addition to the Urbana IX interaction. The $V_{3}^{*}$ uses a pion-exchange between the third particle and the center of mass of the $N N$ system, which in turn uses the full $N-N$ interaction, but only for $T=1$

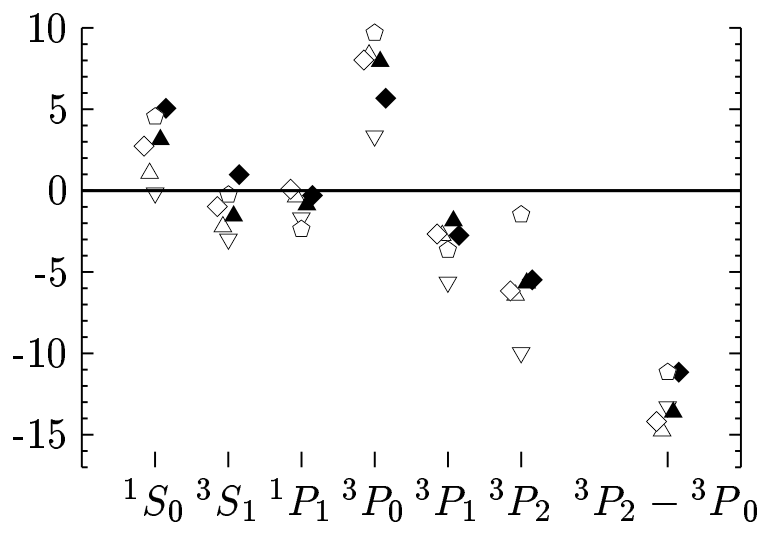

FIG. 7: Deviation of the calculated $p-{ }^{3}$ He phase shifts from the $R$-matrix values in the different channels and the ${ }^{3} P_{2}-{ }^{3} P_{0}$ splitting for different models at $E_{\mathrm{cm}}=4.5 \mathrm{MeV}: \mathrm{A} v_{18}\left(\nabla J_{3}^{+}\right.$ only, $\triangle$ full model), A $v_{18}+\operatorname{Urbana}$ IX $(\boldsymbol{\Delta}), \mathrm{A} v_{8}^{\prime}(\diamond)$ and $\mathrm{A} v_{8}^{\prime}$ $+\operatorname{TLA}(\diamond)$.

and $S=1$. The $V_{3}^{*}$ therefore acts predominantly in the $P$-waves. In order to study the effects of this interaction within a relatively simple operator structure, we approximated the full $t$-matrix by using the central part of the Argonne $v_{18}$ potential as the $N N$-force part in $V_{3}^{*}$, and chose its strength to leave the ${ }^{3} \mathrm{H}$ binding energy unchanged while reproducing the ${ }^{3} P_{2}$ matrix element at $E_{\mathrm{cm}}=2.4 \mathrm{MeV}$.

The full model of the $V_{3}^{*} \mathrm{NNN}$ interaction has only a small influence on the binding energy of ${ }^{3} \mathrm{H}$ [28], which also holds for ${ }^{4} \mathrm{He}$ with the $V_{3}^{*}$ potential in our calculation. However, these operators have a large effect on the $P$-wave phase shifts. If we choose the strength of $V_{3}^{*}$ so that the ${ }^{3} P_{2}$ matrix element is reproduced and the ${ }^{3} \mathrm{H}$ binding remains unchanged, the ${ }^{3} P_{0}$ phase shift 
unfortunately increases again, and therefore part of the improvement due to the larger ${ }^{3} P_{2}$ matrix element is cancelled. Nevertheless, Urbana IX and $V_{3}^{*}$ together achieve an $A_{\mathrm{y}}$ of 0.35 at $E_{\mathrm{cm}}=4.1 \mathrm{MeV}$, a much larger effect than the Urbana IX force alone achieved.

\section{CONCLUSIONS}

We have discussed a new $R$-matrix analysis of the currently available experimental data. The phase shifts calculated in this analysis were compared to an RGM calculation of $p-{ }^{3} \mathrm{He}$ scattering. We showed that realistic $N N$ interactions describe most of the phase shifts quite well but fail to reproduce the ${ }^{3} P_{2}$ and ${ }^{3} P_{0}$ phase shifts. The calculated splitting between these two channels is much too small, and neither the Urbana IX nor the TLA three-nucleon force is able to improve the splitting significantly. In fact, it is more important to include in the calculation negative parity states of the three-nucleon subsystem than one of these two TNIs. These findings show that new contributions to the $N N N$ force acting on the $P$-waves should be considered, like an $L S$ type TNI, as proposed in 29] for the $N-d$ analyzing powers, or the $V_{3}^{*}$ operators proposed in 27].

\section{Acknowledgments}

This work is supported by the DFG (Graduiertenkolleg Erlangen-Regensburg) and the BMBF (contract 06ER926) and used resources at several computer centers (RRZE Erlangen, NIC Jülich, SSC Karlsruhe and LRZ München). We want to thank G. Wellein and G. Hager at the RRZE for their help. The U. S. Department of Energy supported the work of G. M. H. on this study.
[1] H. Witala, D. Hüber, and W. Glöckle, Phys. Rev. C49, R14 (1994).

[2] L. D. Knutson, L. O. Lamm, and J. E. McAninch, Phys. Rev. Lett. 71, 3762 (1993).

[3] A. Kievsky, S. Rosati, W. Tornow, and M. Viviani, Nuc. Phys. A607, 402 (1996).

[4] A. Kievsky and W. Tornow, Proton-Deuteron PhaseShift Analysis above the Deuteron Breakup Threshold, TUNL Progress Report - XXXVIII, TUNL, Durham, USA (1999).

[5] H. M. Hofmann and G. M. Hale, Nucl. Phys. A613, 69 (1997).

[6] D. R. Tilley, H. R. Weller, and G. M. Hale, Nucl. Phys. A541, 1 (1992).

[7] G. M. Hale, D. C. Dodder, J. J. Devaney, and K. Witte, unpublished.

[8] G. M. Hale, D. C. Dodder, J. D. Seagrave, B. L. Berman, and T. W. Phillips, Phys. Rev. C42, 438 (1990).

[9] T. W. Phillips, B. L. Berman, and J. D. Seagrave, Phys. Rev. C22, 384 (1980).

[10] M. T. Alley and L. D. Knutson, Phys. Rev. C48, 1890 (1993).

[11] H. M. Hofmann, in Proceedings of Models and Methods in Few-Body Physics, Lisboa, Portugal 1986, edited by L. S. Ferreira, A. C. Fonseca, and L. Streit (1987), vol. 273 of Springer Lecture Notes in Physics, p. 243.

[12] K. Wildermuth and Y. C. Tang, A Unified Theory of the Nucleus (Vieweg, 1977).

[13] Y. C. Tang, in Topics in Nuclear Physics (Springer, 1981), vol. 145 of Lecture Notes in Physics.
[14] W. Kohn, Phys. Rev. 74, 1763 (1948).

[15] R. B. Wiringa, V. G. J. Stokes, and R. Schiavilla, Phys. Rev. C51, 38 (1995).

[16] B. S. Pudliner, V. R. Pandharipande, S. C. P. J. Carlson, and R. B. Wiringa, Phys. Rev. C56, 1720 (1997).

[17] D. Hüber, J. L. Friar, A. Nogga, H. Witala, and U. van Kolck, nucl-th/9910034 (1999).

[18] C. Winkler and H. M. Hofmann, Phys. Rev. C55, 684 (1997).

[19] A. Nogga, H. Kamada, and W. Glöckle, Phys. Rev. Lett 85, 944 (2000).

[20] A. C. Fonseca, Phys. Rev. Lett. 83, 4021 (1999).

[21] H. Kellermann, H. M. Hofmann, and C. Elster, FewBody-Systems 7, 31 (1989).

[22] T. B. Clegg, A. C. L. Barnard, J. B. Swint, and J. L. Weil, Nucl. Phys. 50, 621 (1964).

[23] M. G. McDonald, W. Haeberli, and L. W. Morrow, Phys. Rev. 133, B1178 (1964).

[24] L. W. Morrow and W. Haeberli, Nucl. Phys. A126, 225 (1969).

[25] Los Alamos physics and cryogenic groups, Nucl. Phys. 12, 291 (1959).

[26] L. N. Katsaurov, R. M. Musaeljan, and V. I. Popov, Atomnaya Energiya, Supplement 5, 71 (1957).

[27] L. Canton and W. Schadow, Phys. Rev. C62, 044005 (2000).

[28] L. Canton and W. Schadow, nucl-th/0006070 (2000).

[29] A. Kievsky, Phys. Rev. C60, 034001 (1999). 